\title{
L'HISTOIRE DE LA NAVIGATION SUR LE DANUBE PARTICULIÈREMENT SUR LE TRONÇON AUTRICHIEN
}

PAR

R. SCHÖRNER *

\section{Le Danube, intermédiaire entre l'Ouest et l'Est}

En étudiant l'histoire de la navigation sur le Danube, on peut constater que, depuis de nombreux siècles, voire mème des milliers d'années, ce fleuve joue un ròle toujours très important de lien entre les pays de l'Ouest et ceux de l'Est. Malgré les hauts et les bas que présentèrent, sur de longues périodes, l'économie et le commerce, en dépit de Iroubles politiques, de guerres, d'attaques à main armée, de disettes ou de catastrophes, et malgre les risques que comporte la navigation en raison des nombreux obstacles, ce fleuve a sans cesse contribué à maintenir la liaison entre l'Ouest et l'Est, et jusqu'à nos jours, le Danube est resté le médiateur entre deux mondes. Cependant, si l'on considere les transformations qui s'annoncent actuellement dans la navigation, et si l'on tient comple d'autres faits, il n'est pas certain qu'il puisse continuer à jouer ce rôle de lien entre les peuples.

\section{La navigation dans la préhistoire et dans l'Antiquité}

(de 5000 arant J.-C. à 500 après J.-C.)

Des decouvertes nous apprennent que déjà à l'époque neolithique, les possibilités qu'offre le Danube en lant que voie de communication furent reconnues et exploitées dans ce sens. Malheureuse-

* Ingenient diplomé, Conseiller de Section auprès de l'Autorito Superteure de la Navigation, Vienne (Autriche). ment, ces découvertes ne nous fournissent aucume indication quant à l'aspect des bateaux qui, à cette époque, naviguèrent sur le Danube. Les objets datant de l'époque entre 1800 et 1000 av. J.-C., objets découverts grâce à de nombreuses fouilles, témoignent qu'en ces temps déjà, et malgré son cours impétueux, le Danube présenta un grand intérêt pour le transport. La première indication écrite nous est donnée, vers 800 avant J.-C., par le poète grec Hésiode, mais ce n'est que trois siècles plus tard que nous trouvons plus de détails sur le Danube. Hérodote, en décrivant une campagne de Darius, roi des Perses, mentionne pour la première fois le nom de Ister en parlant du Danube. De la période allant de 500 à 300 avant J.-C., nous ne savons presque rien, car les quelques découvertes et autres témoignages transmis, ne mettent que peu en lumiere ce que fut la vie sur le fleuve et le long de celui-ci. Il semble, cependant, hors de doute que le commerce de l'ambre ait apporté une certaine animation dans la navigation sur le Danube.

Ce n'est que par les Romains, enfin, que nous obtenons de nombreuses informations sur le Danube, la navigation et le rôle économique que joue cette voie de communication. Grâce à de nombreuses découvertes, il nous est possible de reconstruire assez facilement la vie de cette époque. C'est avant J.-C. même qu'apparaît pour la première fois le nom Danuvius - en allemand Donau - - et l'on sait également que les noms Ister et Danube désignent le même fleuve, fait qui ne semble pas avoir été connu jusqu'à cette époque. Quant aux connaissances sur la longueur du fleuve, ses affluents, son delta et, plus particulièrement, sa source, elles continuent à être assez confuses. Il fut certainement très difficile, à cette époque, de se faire une 


\section{R. SCHÖRNER}

image exacte des dimensions réelles et de la forme effective de la surface du globe terrestre. Il est de fait que les Romains disposaient déjà de cartes géographiques comportant des indications sur les distances, mais l'échelle de ces distances était distordue et les indications concernaient principalement le système routier de l'Empire romain, qui avait pour frontière le Danube, et dont Vindobona - aujourd'hui Vienne _ fut le centre le plus important du trafic et du commerce. Au $\mathrm{II}^{\circ}$ siècle après J.-C., par contre, les connaissances sur le Danube furent certainement déjà assez bonnes, car Pline l'ancien donne, ver's cette époque, une description assez détaillée du Danube, depuis la région où il prend source, en Forêt-Noire, jusqu'à son embouchure dans la mer Noire. Des Romains nous apprenons également qu'ils entretinrent, en permanence, sur le Danube, une flotte de guerre assez importante qui, semble-t-il, était constituée de plusieurs centaines de bateaux, et que, parallèlement a cette navigation armée, leur navigation de commerce fut, elle aussi, très étendue. Parmi les marchandises transportées figurent notamment des briques, des céréales et du fer. (A ce propos, il est certainement intéressant de noter que, tout en ayant culminé au $\mathrm{xr}^{\circ}$ siècle, le transport de céréales joue, de nos jours encore, un rôle important dans la navigation sur le Danube.) Les Romains ont non seulement amélioré les embarcations traditionnelles et simples, et introduit diverses innovations techniques, mais ils ont, avant tout, développé l'organisation de la navigation.

Avec le déclin de l'Empire romain tarissent également les sources d'informations. Nous trouvons par-ci, par-là, encore vers 500 environ après J.-C., quelques indications concernant le voyage qu'effectua une personnalité ecclésiastique ou laïque sur le fleuve, ce qui nous permet de déduire qu'alors, le fleuve servait toujours de voie de communication et de transport; cependant, il est impossible de se prononcer sur l'importance de cette navigation.

\section{La navigation depuis le début du moyen âge jusqu'au commencement de l'ère des machines}

(de 500 à 800 après J.-C.)

Aucune information ne nous est parvenue sur ce que fut la navigation sur le Danube pendant la période de 500 à 800 après J.-C. Pour la première fois, vers l'an 800 l'on indique que, sous le règne de Charlemagne, une flotte était basée sur le Danube, flotte servant de renfort à l'armée qui devait s'opposer à l'invasion des Avares. Ce fut d'ailleurs Charlemagne qui concut le plan de relier, par un canal, le Main au Danube - ce qui, aujourd'hui, semble à peine croyable. Les premiers travaux pour ce projet furent inême entrepris en 793 !

Au cours du $\mathrm{Ix}^{*}$ siecle, le Danube devint la voie de communication vers l'Est. A cette époque, la navigation sur le fleuve fut sans doute assez intense, sans compter les différentes flottes de guerre qui naviguèrent continuellement sur le Danube. En dehors de différentes denrées et des animaux, le sel fut l'un des principaux articles transportés. L'importance du Danube en tant que voie de communication vers l'Est ne cessa de croítre jusqu'au xıI ${ }^{\circ}$ siècle. Avant même que n'eussent lieu les croisades, de nombreux pèlerinages furent entrepris et le trafic s'intensifia progressivement.
A l'époque des croisades, enfin, le Danube devint la plus importante voie de communication vers l'Est et se trouva ainsi placé au premier plan. A la fin du xII" siècle, la ville de Ratisbonne connut son apogée. Les voyages en bateau vers l'Est entrainèrent le commerce avec la Hongrie, la Grèce et le Proche-Orient. Mais, en ces temps, un voyage ou un transport n'était nullement chose courante et rien que le voyage de Ratisbonne à Vienne pouvait se révéler une entreprise dangereuse. Le voyageur de l'époque située entre 1200 et 1500 non seulement se heurtait à de nombreuses dispositions prises par les différents souverains -- dispositions d'ailleurs fréquemment contradictoires — et à des droits de douane souvent illicites et dont furent exonérées certaines catégories de la population, mais il était également impliqué dans de nombreux conflits politiques ou des guerres entrainant souvent le blocage d'une partie du Danube. En plus, de nombreux chevaliers pillards semaient le désordre le long du fleuve en pillant, par exemple, les bateaux de commerce traversant la Valachie. Le «droit d'épave», finalement, conférait au seigneur le droit de s'emparer de l'épave, de toutes les personnes se trouvant à bord, des animaux ou marchandises transportées, et de disposer librement de ces «biens». (En dépit des décisions et interdictions renouvelées à tout moment, et malgré les châtiments dont furent menacés les récidivistes, ce droit fut maintenu avec opiniâtreté jusqu'au xvir siècle.) Sur le lleuve, le manque de sécurité était vraiment très grand. A ces difficultés crées par l'homme, il fallait encore ajouter les obstacles que, de tout temps la nature oppose à la navigation : étiages et crues, poussées de gel, défilés, bas-fonds et rapides, déplacements de matériaux impossibles à évaluer, ruptures de berges non prévues, très fortes vitesses du courant en de nombreux points, maladies et épidémies. Cependant, au mépris de tous ces obstacles, le commerce atteignit alors son plein développement. A partir de 1350 environ, les bateaux remontant le fleuve ne furent plus halés par des hommes mais par des chevaux, procédé qui fut appliqué pendant einq cents ans. Pour haler un bateau il fallait quarante chevaux et même plus, et le voyage de Vienne à Linz - soit une distance d'environ $200 \mathrm{~km}$ - demandait au moins deux semaines!

Au xrve siècle, la position économique des Vénitiens étant dominante, le commerce avec le ProcheOrient régressa et céda la place au commerce intérieur. Vienne devint alors le centre du commerce et de la navigation entre le Danube supérieur et le Danube inférieur, position que la ville put maintenir avec succès pendant de nombreux siècles. Outre le sel, on note parmi les principales marchandises transportées aux $\mathrm{XIV}^{\circ}$ et $\mathrm{Xv}^{\circ}$ siècles, des céréales, du vin, différentes autres denrées, enfin des minerais et des métaux, des peaux, des tissus, des poissons, de la cire et finalement du matériel de guerre. (Au cours des siècles, le sable et les pierres comptèrent également assez souvent parmi les principaux articles transportés sur le Danube.) La navigation connut une vive animation au cours de l'époque de 1500 à 1800 . Les sièges que les Turcs firent par deux fois au xvi" et au xvir siècle, imposèrent un agrandissement continuel de la flotte (même la guerre de Trente Ans fit subir son influence). Vers la fin du xvrr siècle, la flotte impériale d'Autriche compta plus de 5000 bateaux. 
Le commerce avec le Proche-Orient fut complètement arrêté par les guerres contre les Tures et la navigation de commerce dut ètre limitée au cours supérieur du Danube. Par contre, les transports de troupes avancèrent loin en pays hongrois. Au cours de ces campagnes, de nombreuses personnalités royales choisirent le Danube comme route pour effectuer leurs déplacements.

Au xvir siècle débute enfin l'époque où l'on chercha à améliorer le rendement technique de la navigation qui, depuis près de deux mille ans n'avait connu aucune amélioration, se faisait encore par galères ou radeaux et, de ce fait, ne pouvait plus satisfaire des exigences toujours plus grandes. D'autre part, on fit enfin de sérieux efforts pour régulariser le cours du Danube. (A quel point hommes et bêtes devaient-ils ètre torturés par les moustiques, dans cette étendue de prairies et de marécages?) La partie la plus difficile, sur le cours supérieur du Danube est, depuis toujours, le passage du «Struden », près de Grein, qui est l'obstacle le plus important et dont les dangers n'ont été écartés que par la construction de la centrale Ybbs-Persenbeug, et par la retenue ainsi créée qui s'étend jusqu'à Grein, ainsi que par la destruction, il y a quelques années seulement, du seuil appelé «Schwalleck». Nous donnerons plus loin le détail des travaux de régularisation.

$\mathrm{Au} \mathrm{xvIr}^{\circ}$ et au xvir ${ }^{\circ}$ siècle, la navigation est très animée, surtout sur le cours supérieur du Danube, d'une longueur de $800 \mathrm{~km}$ environ, qui s'étend de Ulm, où le Danube devient navigable, jusqu'en Hongrie. A cette époque, la ville de Vienne occupe une position clef. On transporte sur le fleuve, outre les troupes déjà mentionnées et les nombreux voyageurs appartenant à toutes les couches de la population, un grand nombre de marchandises, dont principalement des céréales, du sel, du vin et du bois (radeaux).

Le Xvir ${ }^{\circ}$ siècle est particulièrement important en ce sens que, sous le règne de Marie-Thérèse, les pays danubiens de l'Est devinrent accessibles et, partant, mis en valeur.

\section{La navigation \\ du début de la force à vapeur à nos jours}

(à partir de 1800)

On peut considérer le 17 septembre 1830 comme étant la date de naissance de la navigation à vapeur sur le Danube. C'est, en effet, ce jour-là qu'eut lieu le voyage d'essai du premier bateau de ce genre, le «Franz-I», qui parcourut en $14 \mathrm{~h}$ les $300 \mathrm{~km}$ séparant Vienne de Budapest, et mit $48 \mathrm{~h}$ pour effectuer le voyage en sens inverse. Ce voyage fut réalisé vingt-trois ans après le premier voyage du tout premier bateau à vapeur - le voyage de New York à Albany, qu'effectua, en 1807, le «Clermont» de Fulton - et un an après la fondation, en Autriche, de la «Erste DonauDampfschiffahrts-Gesellschaft (I.DDSG), la première Association pour la Navigation à Vapeur sur le Danube, qui était également propriétaire du «Franz-I». Ta I.DDSG occupa une position de monopole, position conservée jusqu'en nos jours. En raison de cette position de monopole, l'évolution de la navigation sur le Danube, au $\mathrm{xIx}^{\circ}$ et au $\mathrm{xx}^{\circ}$ siècle, et l'évolution de l'Association pour la Navigation à Vapeur sur le Danube sont parallèles. Il suffit donc de suivre les fluctuations subies par cette société pour être informé de l'importance de la navigation à cette époque.

Au moment de la fondation de la I.DDSG il n'y a encore ni chemin de fer, ni téléphone, ni télégraphe; le fleuve lui-même n'a pratiquement subi aucune régularisation et, en raison des nombreux obstacles s'opposant à la navigation, il semble douteux que des bateaux plus importants et alourdis aussi par leurs machines, puissent néanmoins présenter un tirant d'ean suffisamment faible pour

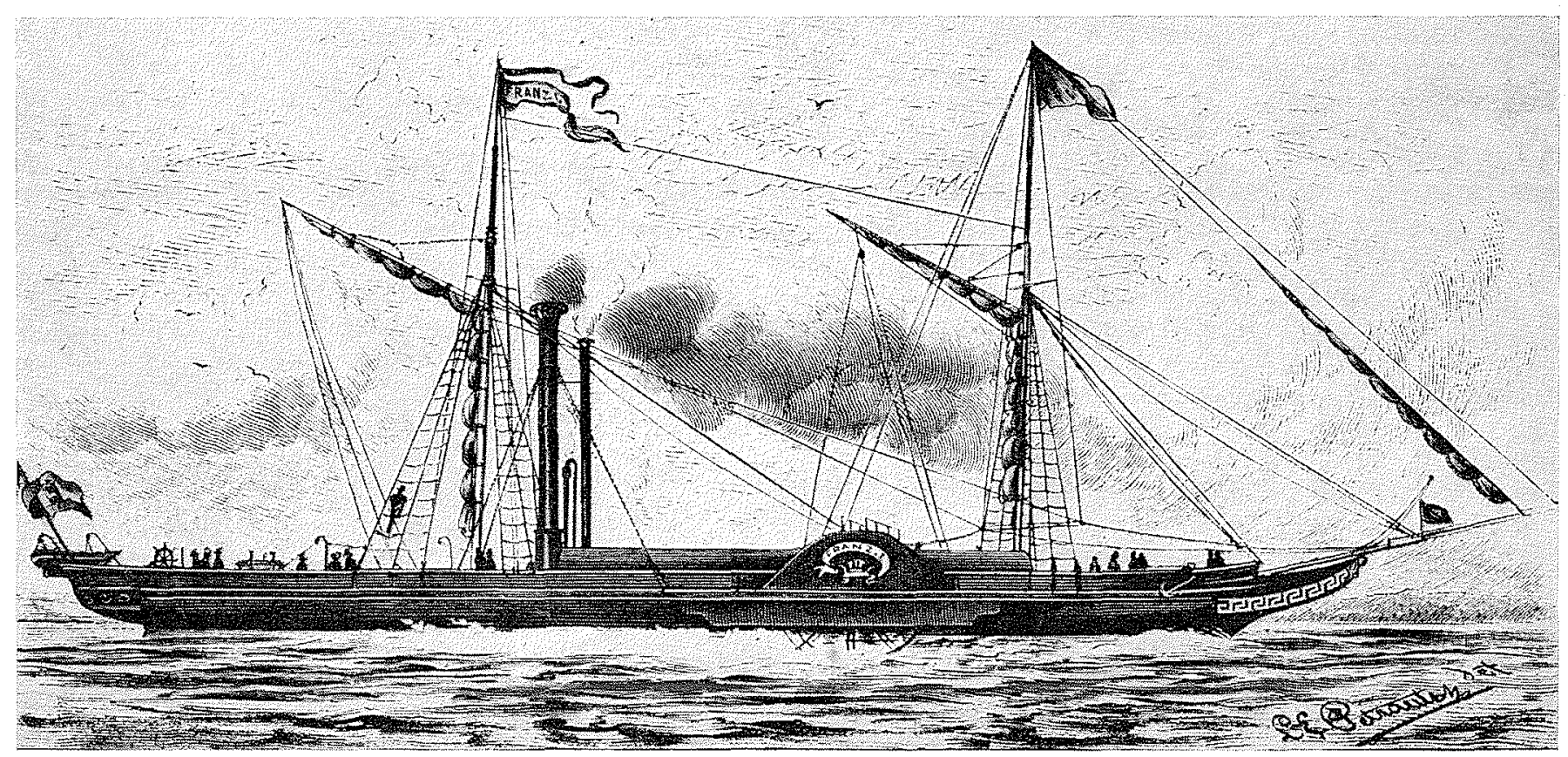

1/ « Franz I », le premier bateau de Ia I.DDSG, construit en 1830.

Das erste Dampischiff der Donaudampfschiffahrtsgesellschaft, « Franz I », erbaut im Jahre 1830. 


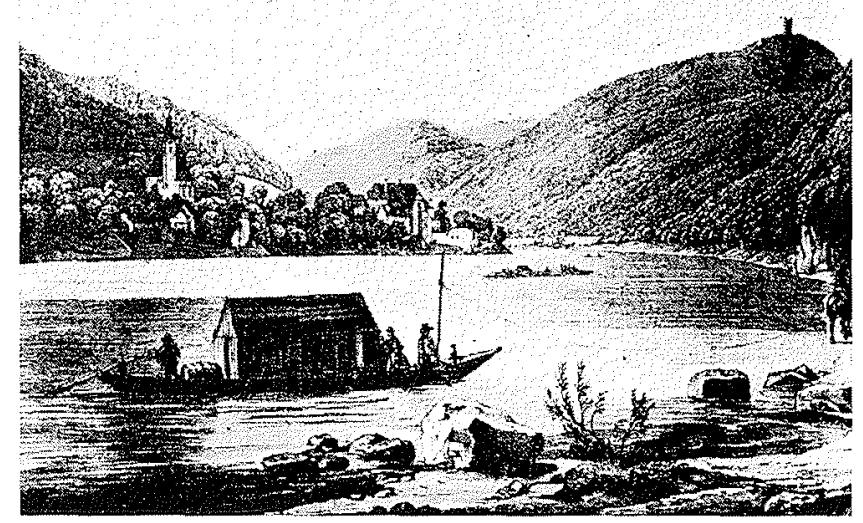

2/ Cette lithographie de Jacob Alt représente l'un des bateaux, portant le nom étrange de «Flieszstein ». Ces bateaux servirent surtout au transport des voyageurs vers l'amont. sur le cours supérieur du Danube. Ce mode de transport fut appliqué depuis le xvi" siècle jusqu'au début du xx" siècle (t). Die von Jacob Alt verfertigte Lithographie zeigt eines der mit dem eigenartigen Namen «lieszstein $\gg$ bezeichneten Schiffe, die hauptsächlich der Personenbeförderung stromaufuärts alu der oberen Donau dienten und bom 17.Jht. bis zu Anfang des 20 . Jhts (!) verkehrten.

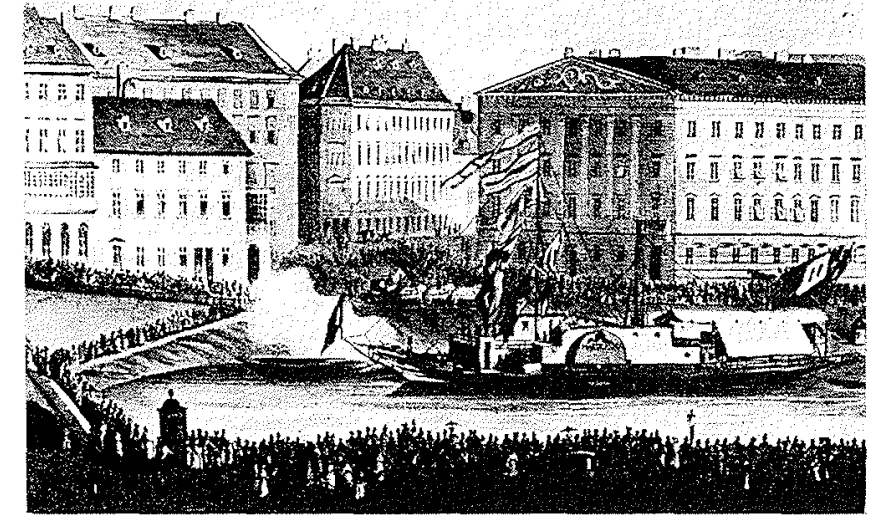

3/ La « Maria Anna », bateau pour passagers, lors de son premier voyage, de Vienne à Linz, en septembre 1837.

Erste Fahrt des Personendamfers « Maria Anna $»$ bon wien nach Linz, September 1837. pouvorr naviguer sur le fleuve. Mais la décision est prise; cependant, sa réalisation demande beaucoup de courage et d'énergie et aussi une bonne dose de patience. En tenant compte d'un certain nombre de considérations, l'entreprise se décide pour la navigation sur la partie est du Danube car, d'une part, le trafic sur le cours supérieur du Danube est, depuis longtemps, « rodé »; d'autre part un bateau naviguant à la vapeur est particulièrement indiqué pour assurer le transport sur de longues distances et, finalement l'intérêt que l'on porte aux Etats danubiens de l'Est, s'est nettement accru en raison de la nouvelle politique économique européenne. En peu de temps, il est possible de développer l'organisation à tel point que la liaison par bateaux peut être étendue jusqu'au delta, et au-delà jusqu'à Constantinople et Smyrne. Quant au tronçon de Vienne vers l'amont, de nombreux obstacles rendent la navigation très difficile, même pour des bateatlx à vapeur. Ce n'est que sept ans après le royage du vapeur «Franz-I» que le vapeur «Maria-Anna» se rend de Vienne à Linz, et il met plus de $55 \mathrm{~h}$ pour effectuer ce voyage. Peu de temps après, le premier vapeur de la société allemande «Königlich bayrischwürttemhergische Donaudampfschiffahrtsgesellschaft» (Société de Navigation à Vapeur des Royaumes de Bavière et du Wurtemberg), venant de Ratisbonne, arrive à Linz. L'année d'après, en 1838, les deux sociétés créent un service régulier et, pour la première fois, des bateaux propulsés par des machines relient Ratisbonne au delta. Dans la même année, la société autrichienne sépare le transport des voyageurs du transport des marchandises.

La navigation sur le Danube prend par la suite un essor insoupconné. La liaison entre Ulm et Sulina devient possible grâce à des améliorations du chenal navigable, à la création de nouveaux embarcadères et de nouvelles installations aux points d'accostage, à une amélioration apportée dans l'organisation, à la création d'un centre de formation pour les équipages et grâce aussi à l'ex- ploitation de mines fournissant un charbon approprié.

Comme nous l'apprend son histoire, la société mène, depuis le jour de sa fondation jusqu'à nos jours, une lutte perpétuelle contre un grand nombre d'obstacles fâcheux. La navigation était paralysée par des troubles politiques et des guerres, des catastrophes laissaient des traces, des diseltes dans les pays de l'Est ou des récoltes trop riches dans les pays occidentaux avaient une incidence fâcheuse sur le principal article de transport, les céréales. De même, plusieurs épidémies de choléra sévissant sur le cours inférieur du Danube, contraignirent à des restrictions. De plus, la société se heurta continuellement à des difficultés créées par les différentes administrations de l'Est. Comme par le passé, de nombreux obstacles naturels constituaient des dangers, notamment les Portes de Fer qui, sur le cours inférieur, menaçaient particulièrement la navigation. Mais la sociétẻ surmonta un à un tous ces obstacles et vers 1890 , elle put être fière de son bilan : elle possédait 200 bateaux d'une puissance de $72000 \mathrm{ch}, 800$ chalands pouvant transporter $300000 \mathrm{t}$ et environ 100 bateaux spéciaux tels que pontons, grues, ateliers flottants, grues à vapeur flottantes, élévateurs de grains, etc. Vers la fin du $\mathrm{xrx}^{*}$ siècle, la société rayonnait sur un réseau de près de $5700 \mathrm{~km}$ qui comprend également les affluents. Elle était ainsi la plus importante entreprise de navigation intérieure du monde entier, jouissait d'une considération internationale et elle avait une influence décisive sur tout ce qui concerne la partie navigable du Danube. Les dernières décennies du xix" siècle apportèrent une amélioration par introduction du halage par chaînes. Ce procédé fut appliqué sur différentes parties du Danube et se maintint jusqu'en 1906. Pendant la même période, plusieurs obstacles furent supprimés dans les cours supérieur et inférieur du Danube. Au cours des années 1900 à 1914, les transports se chiffrent par plus de 2 millions de personnes et plus de 2 millions de tonnes de marchandises par an. 
Au cours de la première guerre mondiale, la société doit satisfaire à un maximum d'exigences, mais elle sait $y$ faire face en faisant les plus grands efforts et en mobilisant toutes ses réserves. Après la première guerre mondiale, alors que la monarchie austro-hongroise est en décadence, les conditions de paix désastreuses, dont les répercussions se font sentir encore de nos jours, lui font perdre près de la moitié de sa flotte. Sans pouvoir prétendre à un dédommagement, elle doit céder la plupart de ses unités à la Tchécoslovaquie, à la Yougoslavie, à la Roumanie et à la France (!). Son réseau d'exploitation se réduit à un quart de ce qu'il fut avant la guerre; il ne s'étend plus que sur $1300 \mathrm{~km}$ environ. Sur le cours inférieur du Danube, les sociétés nationales de navigation fondées antérieurement en Hongrie, en Serbie, en Roumanie, en Bulgarie et en Russie, succèdent à la Société autrichienne de Navigation à la vapeur. Vers 1920, pour la premiere fois dans l'histoire du Danube, des bateaux-citernes transportent d'importantes quantités de pétrole brut et de ses dirivés, depuis la Roumanie vers l'amont. La navigation qui, depuis l'effondrement de la monarchie, est complètement paralysée, s'anime de nouveau peu à peu. Cependant, conformément au régime international établi par le traité de Paris, ce ne sont plus seulement les sociétés nationales des Eiats danubiens qui participent à la navigation sur tonte la section fluviale comprise entre Ulm et le delta, mais il y a également des bateaux naviguant sous les drapeaux anglais, hollandais et français. Cependant, la concurrence entre ces différentes sociétés ainsi que celle du chemin de fer sont très lortes, et chacune de ces sociétés subit de grosses pertes. Ce sont précisément ces pertes qui, en fin de compte, permettent à la sagesse humaine de lemporter: les entreprises de navigation se réunissent en un "groupement d'exploitation» qui poursuit le but d'une meilleure exploitation et t'une meilleure utilisation de la capacité de chargement. Ce groupement est maintenu jusqu'à la fin de la deuxième guerre mondiale.

Les pertes qu'éprouve la société pendant la deuxieme guerre mondiale, dépassent encore de beaucoup celles subies pendant la guerre de 19141918. Elle perd ainsi, soit par la guerre, soit par les conditions de paix de nouveau imposées, près de 360 bateaux, chalands et bateaux-citernes, une centaine de bateaux spéciaux et tous les biens qu'elle possède à l'étranger et qui comprend outre des chantiers de construction et de nombreuses installations d'accostage, avant tout, les grandes mines de charbon de Fünfkirchen.

Au cours des années succédant à la deuxième guerre mondiale, la I.DDSG ainsi que la « Continentale Motorschifrahrtsgesellschaft A.G. (COMOS) (Société continentale de Navigation à Moteur, S.A.) qui, en 1923, s'est établie à Vienne, ne sont guère favorisées par les conditions atmosphériques, très mauvaises; les étiages et crues, le charriage de glace, imposent à plusieurs reprises une restriction ou mème une interruption complète de la navigation. Les bateaux à vapeur d'ancienne construction sont transformés et adaptés pour marcher à l'huile. Pour le transport des marchandises sur le Danube, les entreprises autrichiennes disposent d'environ : 40 remorqueurs d'une puissance d'environ $33000 \mathrm{ch} ; 240$ chalands marchands d'une capacité de transport d'environ $190000 \mathrm{t}$; et, de 70 baleaux-citernes d'une capacité de transport d'environ $70000 \mathrm{t}$.

Les principales marchandises sont classées par ordre de quantités transportées:

- huiles minérales et produits d'huile minérale,

- charbon,

- fer et acier,

- minerai,

céréales, et

- engrais.

Le transport des vovageurs sur la partie autrichienne du Danube, partie d'une longueur de $350 \mathrm{~km}$ environ et comprise entre Passau et Hainburg, est assuré par 10 bateaux d'une puissance de $6200 \mathrm{ch}$ environ et d'une capacité de transport de 9000 personnes.

La position dominante acquise par la I.DDSG au cours du $x_{1} x^{*}$ siècle, est, hélas, perdue à tout jamais. La division matheureuse de l'Europe et l'inévitable modification de la structure économique d'une part, la mise à disponibilité d'autres sources d'énergie et d'autres moyens de transport d'autre part, ne laissent que peu d'espoir que la navigation autrichienne puisse un jour reconquérir une place plus importante. Il serait donc vain de pousser plus loin nos considérations.

\section{Bibliographie}

[1 Dr Lujo Toncic-Sonns: Die Enteckung der Donau (La découverte du Danube). Zeitschrift Vniversum, Helt 1 , 5 Jahrgang, Seite $17 \mathrm{ff}$, Wien, Jänner 1950.

[2] Dr Ernst NFwerLowsky: Die Schiffahrt und Flössere im Raume der oberen Danau (La navigation par bateaux et radeaux dans la région du haut Danube). Oberösterreichischer Landesverlag, Ling, 1952, 1954 et 1963.
[3] Festschrift der I.DDSG zum 125-jührigen Bestand des Cnternehmens, (Edition commémoratrice de la I.DISSG à l'oceasion du 125" anniversaire de l'entreprise) Wien, 1954.

Les photos extraites des arehives de la I.PDSG ont été aimablement mises à notre disposition par cette dernière.

\section{Zusammenfassung \\ Die geschichliche Entwicklung der Schiffahrt auf der Donau Eine kurze Darstellung mit vornchmlicher Betrachtung der österreichischen Donaustrecke Von Dipl. Ing. Robert Schörner*}

Der Yerfasser beschreibt einleitend die Funktion der Donau als Vermittler zwischen West und Ost und beleuchtet die Zukunftsaussichten für ihre völkerverbindende Rolle.

Es wird in weiteren ein Ceberblick gegeben über die Schiffahrt auf diesen Strom in der vorgeschichtlichen Zeit und im Altertum (5000 v. Chr. bis $500 \mathrm{n}$. Chr.). Insbesondere wird die Schiffahrt der Römer behandelt auch in Bezug auf Handels- und Kriegsschiffahrt sowie die Art der Transportgüter (Ziegel, Getreide, Eisen).

* Sektionsrat Dipl. Ing., Oberste Schiffahrtsbehörde, Wien (Oesterreich). 


\section{R. SCHỎRNER}

In dem Zeitabschnitt von 500 bis $1800 \mathrm{n}$. Chr. - also etwa vom Beginn des Mittelalters bis zum Anbruch des Maschinenzeitalters - zeichnet sich immer deutlicher die Bedeutung des Stromes als Verkehrsweg nach dem Osten (naher Orient) ab. Damals wurde schon die erste Planung für einen Main-Donau-Kanal vorgenommen. Schriffreisen um etwa $1300 \mathrm{n}$. Chr. wurden nicht nur durch naturbedingte Schwierigkeiten sondern auch durch das "Strandrecht" u.a. sehr erschwert. Durch das Einführen des Pferdezuges zum Ziehen der Schiffe stromaufwärts ergab sich eine wesentliche Steigerung des Anteiles des Schiffahrtstransportes im Rahmen des Binnenhandels. Im 14 Jht. war Wien Handelszentrum und Verkehrsknotenpunkt. Der Schiffahrstransport umfaßte vor allem Salz, Getreide, Wein, Erz, Textilien und Steine. Versuche die naturbedingten Schwierigkeiten für die Stromschiffahrt zu beseitigen wurden bereits im 18. Jht. unternommen. Wien beherrschte damals in einer Schlüsselstellung das Schiffahrtsgeschehen am Donaustrom. Durch Maria Theresia wurden mit Hilfe des Stromes die östlichen Donaustaaten erschlossen und diese Gebiete einer Kultivierung zugeführt.

Vom Beginn des "Dampfmaschinen-Zeitalters" (etwa ab 1800) zeichnet sich ein weiterer, wesentlicher Zeitabschnitt in der Entwicklung der Donauschiffahrt ab. Es wurde die I. DDSG (Erste DonaudampfschiffahrtsGesellschaft) gegründet und bald darauf erfolgte die erste Fahrt eines Dampfschiffes von Wien stromab-und stromaufwärts. Der Fahrgast- und Güterverkehr erstreckte sich alsbald von Regensburg bis zur Donaumündung bzw. bis Konstantinopel und Smyrna. Die I. DDSG erfährt eine Ausweitung zum großten Binnenschiffahrtsunternehmen der Welt mit einem Betriebsnetz von $5700 \mathrm{~km}$ Länge.

Es werden im weiteren die Transportleistungen der I. DDSG bis zum 1. Weltkrieg und die Auswirkungen dieses Krieges auf die Donauschiffahrt - insbesondere durch die Gründung nationaler. Schiffahrtsgesellschaften im Unterlauf der Donau - aufgezeigt. Ebenso werden die Verluste der I. DDSG nach dem 2. Weltkrieg, der gegenwärtisge Stand der Donauflotte im Verein mit der COMOS (Continentale Motorschiffahrtsgesellschaft- AG), sowie das Ausmaß des Massentransportes und der Personentransport auf der österreichischen Donaustrecke (FahrgastSchiffe) behandelt.

Die Schlußbetrachtung befaßt sich mit einem Ausblick auf die Zukunftsaussichten für den Schifrahrtsverkehr auf der Donau.

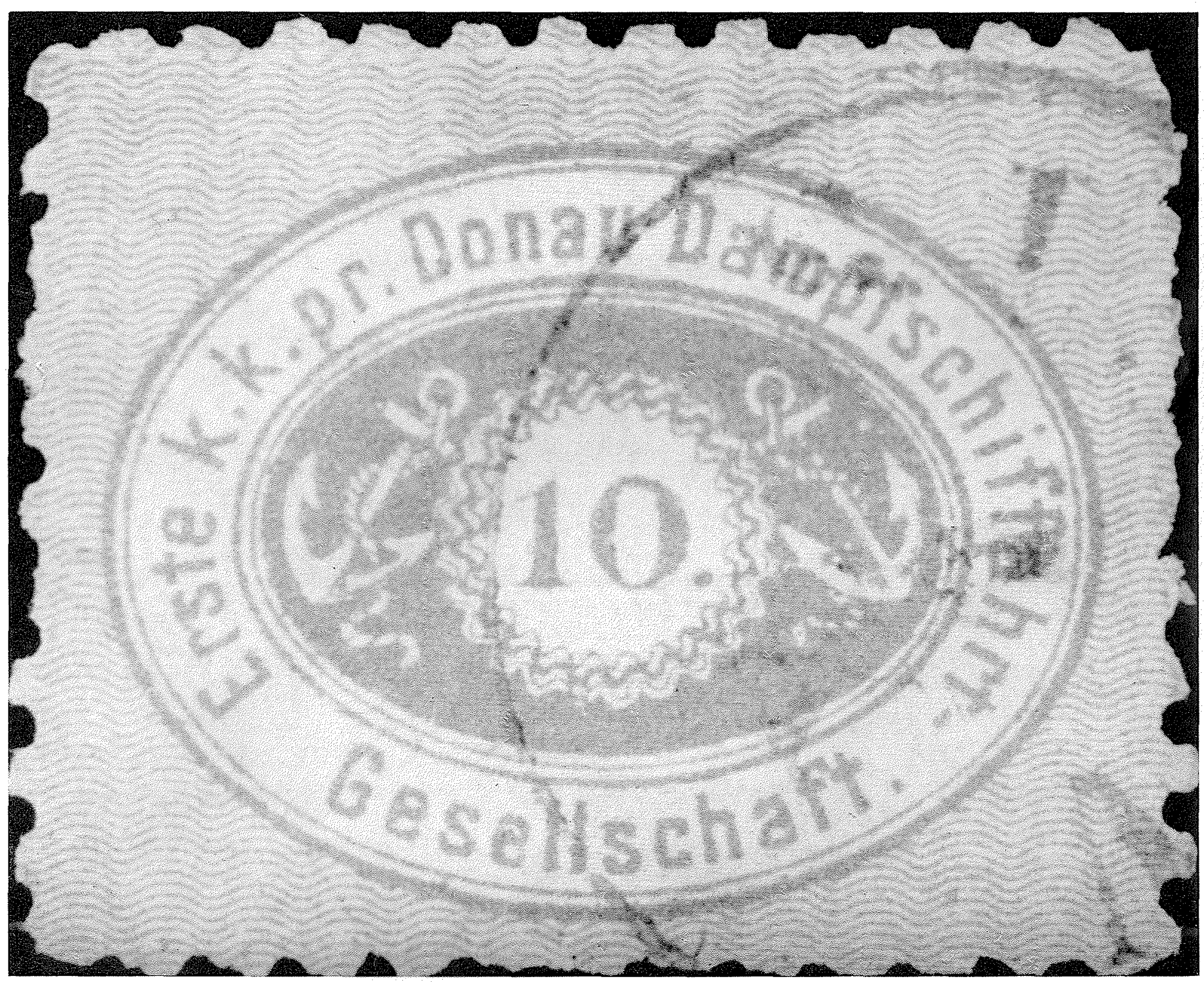

4/ Timbre émis par la « Donaudampeschiffahtsgesclischaft » pour le service postal sur le Danube en Roumanie, Bulgarie et Serbie. Ce timbre fut employé de 1866 à 1880.

Briefmarke der Donaudampfschiffalutsgesellschaft fïr den Postdienst auf der Donau in Rumänien, Bulgarien und Serbien, welche von 1866 bis 1880 in Verwendung war. 